\title{
Treatment outcomes of ureteral stenting for malignant extrinsic ureteral obstruction: a comparison between polymeric and metallic stents
}

\author{
Jumpei Asakawa \\ Taro Iguchi \\ Satoshi Tamada \\ Noriko Ninomiya \\ Minoru Kato \\ Takeshi Yamasaki \\ Tatsuya Nakatani \\ Department of Urology, Osaka \\ City University Graduate School of \\ Medicine, Osaka, Japan
}

This article was published in the following Dove Press journal: Cancer Management and Research

Purpose: To compare treatment outcomes, more specifically patency rate, of polymeric and metallic stents for malignant ureteral obstruction.

Patients and methods: Between August 2007 and September 2017, we retrospectively analyzed the data of 92 patients (126 ureters) having a diagnosis of malignant extrinsic ureteral obstruction treated with indwelling ureteral stents (polymeric and full-length metallic stents). Of these patients, 35 (54 ureters) were treated with polymeric stents and 57 (72 ureters) with a Resonance ${ }^{\circledR}$ metallic stent. The observation period was censored to 1 year. Survival rate in cases of malignant ureteral obstruction was calculated, and the relationship between the causes of ureteral obstruction, the stent type, and the patency rate was evaluated.

Results: The median observation period was 145 days, with a median survival time of 258 days. The stent patency rate was $70.9 \%$ at 1 year, regardless of stent type. Stent occlusion was observed in 20 patients (33 ureters). According to stent type, occlusion of the polymeric and metallic stents was identified in $12(22 \%)$ and $8(11 \%)$ cases, respectively. The clinical features associated with stent failure were assessed. In univariate analysis, the patency rate was significantly better for the metallic stent than for the polymeric stent (1-year patency rate; $78.4 \%$, $61.1 \%$, respectively, HR, 2.15; 95\% CI, 1.07-4.33; $p=0.031$ ). However, the patency rate among patients with abdominal dissemination, lymph node metastasis, and direct compression by tumor was not significantly different.

Conclusion: Indwelling ureteral stents, particularly metallic stents, are effective for the treatment of malignant ureteral obstruction.

Keywords: metallic stent, malignant ureteral obstruction, polymeric stent

\section{Introduction}

Extrinsic ureteral obstruction refers to an external physical obstruction of the ureter caused by the direct invasion of gastrointestinal and gynecologic cancer, lymph node metastasis, peritoneal dissemination, and benign diseases, such as myoma uteri and retroperitoneal fibrosis. Malignant ureteral obstruction (MUO) has been reported to be an indicator of poor prognosis, with a median life expectancy of $<1$ year among patients with metastatic cancer causing ureteral obstruction. ${ }^{1,2}$ Conventional treatment for extrinsic ureteral obstruction includes securing the urinary tract using a polymeric ureteral stent or creation of a nephrostomy. Jeong et $\mathrm{al}^{1}$ evaluated the outcomes of a ureteric polymeric stent for nonurological MUO, reporting a stent failure rate of

Correspondence: Satoshi Tamada Department of Urology, Osaka City University Graduate School of Medicine, I-4-3 Asahi-machi, Abeno-ku, Osaka 5458585, Japan

Tel +81666453857

Fax +8I 666474426

Email s-tamada@med.osaka-cu.ac.jp 
$16 \%$, with a median interval of 3.2 months, from the first stent insertion to failure, and patients requiring, on average, 1.9 stent replacement prior to death. As polymeric stents are fragile against extrinsic MUO, they often require replacement. Therefore, several studies have evaluated the applicability of metallic stents, rather than polymeric stents, for the treatment of MUO. ${ }^{3-5}$ In 119 cases of extrinsic MUO treated with metal mesh stents, Liatsikos et $\mathrm{al}^{6}$ reported the following adverse effects: hyperplastic reaction, encrustation and tumor ingrowth (45 cases); secondary intervention (17 cases); and stent migration (13 cases). Considering the rate of adverse effects and a primary patency rate of only $51.2 \%$, they concluded that metal mesh stents have limited use in the treatment of extrinsic MUO. Moreover, metal mesh stents are short and semi-permanent and, therefore, difficult to remove. An ideal stent should be easy to implant, maintain ureter patency with no additional interventions, allow longer intervals between stent exchange, and be easy to remove. ${ }^{4}$ Therefore, a new design for a full-length metallic ureteric

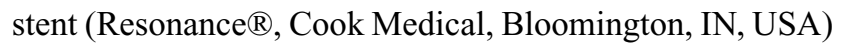
has been developed for the management of MUO to overcome some of the limitations of polymeric or metallic stents.

Since Borin et $\mathrm{al}^{7}$ reported on their initial experience with metallic stents, metallic stents have been approved for clinical use in many countries since 2007 , and have been available in Japan since December, 2014. Although several reports of adverse outcomes of metallic stents have been published, ${ }^{8-13}$ these case series have generally included a small number of patients.

In a previous study, we reported the treatment outcomes of metallic stents for extrinsic MUO in a relatively large retrospective cohort of 52 cases (66 ureters). ${ }^{14}$ Among this study cohort, the stent patency rate was $86.0 \%$ at 6 months and $60.0 \%$ at 1 year. Nephrostomy was required in 8 cases (15.4\%), due to stent failure. In addition, the occlusion rate was significantly higher for bilateral than unilateral ureteral obstruction. Although we concluded that full-length metallic stents could be the first treatment choice for MUO, our study was limited by the inherent bias of a retrospective design and, therefore, a direct comparison of the treatment outcomes of metallic and polymeric stents was not possible. Only 1 previous study has compared the treatment outcomes of polymeric and metallic stents, ${ }^{15}$ indicating that metallic stents provided better patency than polymeric stents. However, in this previous study, a metal mesh stent, instead of the Resonance ${ }^{\circledR}$ stent (full-length metallic stent) was used. Therefore, in the present study, we aimed to retrospectively review the treatment outcomes for the ureteral stents in 92 patients (126 ureters) with extrinsic MUO, and, for the first time, compare the treatment outcomes of polymeric and full-length metallic stents.

\section{Patients and methods}

Between August 2007 and September 2017, ureteral stents (polymeric and full-length metallic stents) were implanted in 92 patients (126 ureters) diagnosed with malignant extrinsic ureteral obstruction, and their data were retrospectively analyzed. Before metallic stent was approved in Japan, we inserted polymeric stents for patients with MUO. From 2014 onward, we inserted metallic stents for these patients. Among 92 patients with MUO, 35 (54 ureters) were treated with polymeric stents and 57 (72 ureters) with the Resonance ${ }^{\circledR}$ (Cook Medical) metallic stent. The polymeric stents were exchanged every 3-6 months. We observed these patients for $>1$ year; however, no more cases were observed as having stent occlusion after 1 year and based on the recommendation of a 1-year indwelling period for metallic stents, and so the observation period for our study was censored to 1 year. The clinical characteristics of the study patients are shown in Table 1 .

In our hospital, all stents were placed in a standard retrograde manner by using X-ray guidance under local or spinal anesthesia. Follow-up imaging, blood sampling, and abdominal ultrasound examination were performed on postoperative day 1 and at a 3-or 6-month interval in stable patients. Primary stent patency was defined as complete or partial resolution of hydronephrosis within 1 week. Partial resolution of hydronephrosis was defined as a decrease in hydronephrosis, according to the Ellenbogen classification. ${ }^{16}$ Stent failure was defined as unanticipated stent exchange or nephrostomy placement due to signs or symptoms of recurrent ureteral obstruction (recurrent hydronephrosis or increasing serum creatinine). ${ }^{12}$

Differences in clinicopathological variables and primary patency rate between metallic and polymer stents were analyzed using a chi-squared analysis. Survival rate, among cases of malignant ureteral obstruction, was calculated using the Kaplan-Meier method. The log rank test was used to evaluate the relationship between the stent type and the patency rate. Cox proportional stepwise multivariate analysis was used to evaluate the association between the causes of ureteral obstruction, stent type, and stent failure.

All $p$-values were 2 -sided, and a $p<0.05$ was considered statistically significant. Statistical analysis was performed using Microsoft Excel ${ }^{\circledR}$ (Microsoft, Redmond, WA, USA).

Permission to access the database for review of the medical records of these patients was approved by the local 
Table I Patient characteristics

\begin{tabular}{|c|c|c|c|c|c|c|c|}
\hline \multirow[b]{2}{*}{ Total number of ureters } & \multicolumn{2}{|c|}{ All patients $(\mathrm{N}=92)$} & \multicolumn{2}{|c|}{ Polymeric $(\mathbf{N}=35)$} & \multicolumn{2}{|c|}{ Metallic $(\mathbf{N}=57)$} & \multirow[t]{2}{*}{ p-value } \\
\hline & 126 & & 54 & & 72 & & \\
\hline Age (median) (years) & 66 & $(24-90)$ & 64 & $(35-80)$ & 68 & $(24-90)$ & 0.176 \\
\hline Sex & Male: 39 & Female: 53 & Male: II & Female: 24 & Male: 27 & Female: 45 & 0.538 \\
\hline Observation period (median) (days) & 145 & $(1-365)$ & 178 & $(2-365)$ & 163 & $(2-365)$ & 0.244 \\
\hline Primary disease & & & & & & & 0.002 \\
\hline Gastrointestinal cancer & 50 & & 13 & & 37 & & \\
\hline Gynecological cancer & 31 & & 20 & & 11 & & \\
\hline Urogenital cancer & 4 & & 0 & & 4 & & \\
\hline Other & 7 & & 2 & & 5 & & \\
\hline Obstructed side & Unilateral 58 & Bilateral 34 & Unilateral 16 & Bilateral 19 & Unilateral 42 & Bilateral I5 & 0.007 \\
\hline Cause of ureteral obstruction & & & & & & & 0.111 \\
\hline Direct compression by tumor & 30 & & 11 & & 19 & & \\
\hline Lymph node metastasis & 26 & & 14 & & 12 & & \\
\hline Abdominal dissemination & 36 & & 10 & & 26 & & \\
\hline
\end{tabular}

research ethics committee at Osaka City University (approval number 3440). A waiver for individual patient consent for this retrospective study was also obtained from this ethics committee. All clinical data were anonymized.

\section{Results}

Patient characteristics are summarized in Table 1. The prevalence rate of obstruction due to gastrointestinal cancer and of bilateral obstruction was higher among the patient group in whom a metallic stent was used than among the patient group in whom polymer stents were implanted. The median observation period was 163 days (range, 2-365 days). The median survival time for the 92 patients in our study cohort was 258 days (Figure 1). The stent patency rate was $70.9 \%$ at 1 year, regardless of stent type (Figure 2). Stent occlusion within 1 week after indwelling stent was observed in 10 patients (18 ureters); the stent patency rate was $85.7 \%$ (polymeric stent $79.6 \%$, metallic stent $90.3 \%, p=0.091)$. Occlusion was identified in $12(22 \%)$ cases in the polymeric stent group and $8(11 \%)$ of cases in the metallic stent group, over the entire observation period. Stent occlusion was observed in 20 patients ( 33 ureters): 10 of the 31 patients with gynecological cancer $(32 \%) ; 7$ of the 50 patients with gastrointestinal cancer (14\%); 2 of the 4 patients with urogenital cancer $(50 \%)$; and 1 of the 7 patients with "other" cancers. With regard to the cause of stent occlusion, lymph node metastasis was identified in 7 ureters (19\%), tumor compression in $6(16 \%)$, and peritoneal dissemination in $14(26 \%)$.

In the statistical analysis to identify the clinical features associated with stent failure, the patency rate was not significantly different among patients with abdominal dissemination, lymph node metastasis, and direct compression by tumor (Table 2). On univariate analysis, the patency rate was found to be higher for metallic than polymeric stents

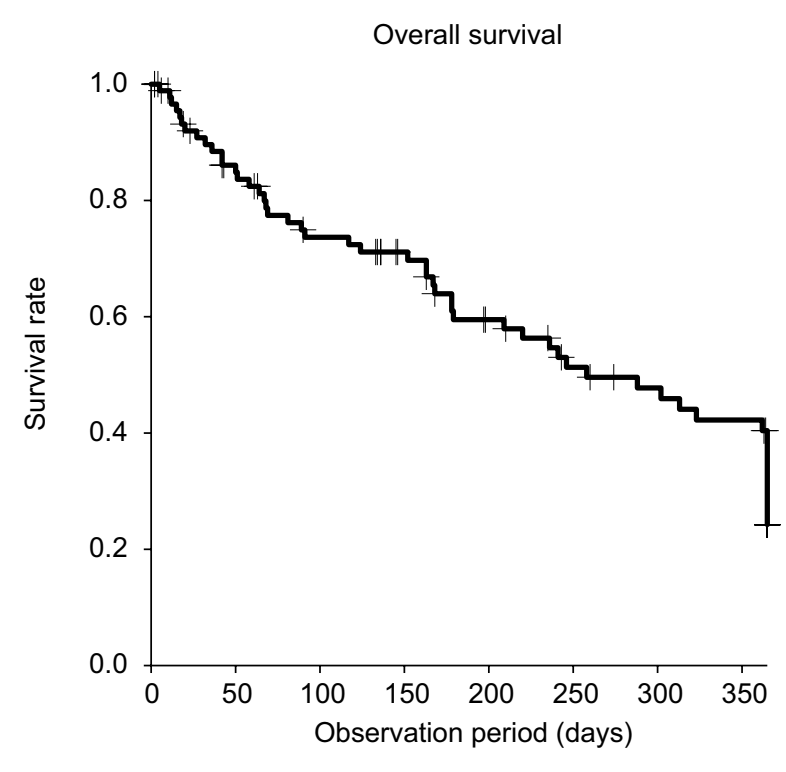

$\begin{array}{lllllllll}\text { No. at risk } & 92 & 71 & 59 & 51 & 39 & 31 & 26 & 20\end{array}$

Figure I Median survival time of the 92 patients with MUO

Abbreviation: MUO, malignant ureteral obstruction.

(1-year patency rate; $78.4 \%$ and $61.1 \%$, respectively; HR, 2.15; 95\% CI, 1.07-4.33; $p=0.031$; Figure 3). However, on multivariate analysis, no significant between-group difference was identified.

\section{Discussion}

Currently, metallic stents are as commonly used as polymeric stents; however, no previous study has compared the treatment outcomes of these 2 different stent types. Therefore, this study is the first to retrospectively compare the treatment outcomes for polymeric and metallic stents, providing evidence that metallic stents are superior to polymeric stents in improving the patency rate against MUO. 
MUO has been reported to be an indicator of poor prognosis, with the median life expectancy being $<1$ year in patients with metastatic cancer causing ureteral obstruction. ${ }^{1,2}$ Polymeric double J stents or nephrostomy have been the most common treatment for ureteral obstruction in these patients. However, the problem of a high occlusion rate in polymeric stents has yet to be resolved. ${ }^{17-21}$ Furthermore, polymeric stents and nephrostomy tubes require regular replacement. Considering the short life expectancy of these patients, avoiding stent exchange is preferable to prevent unnecessary complications or cosmetic changes. Among our 92 patients, the median survival time was 258 days, with only 19 patients (20.7\%) surviving beyond 1 year. Therefore, the higher patency rate provided by metallic stents, with an anticipated 1-year indwelling period, would be beneficial for the treatment of MUO in this clinical population.

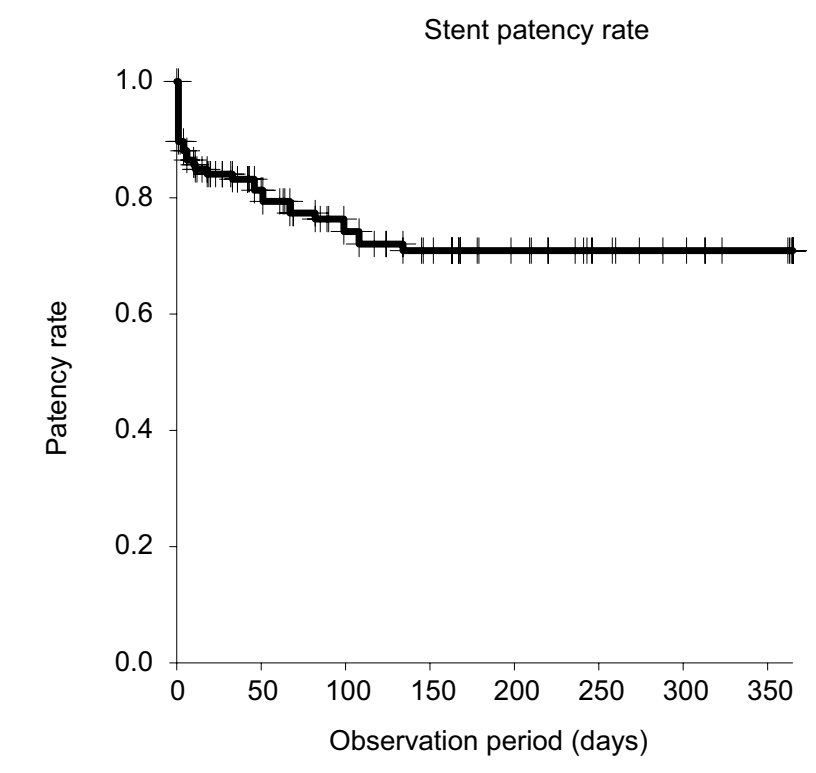

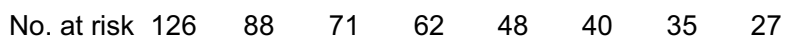

Figure 2 Stent patency rate for MUO.

Abbreviation: MUO, malignant ureteral obstruction.
In our previous study evaluating the treatment outcomes of indwelling metallic stents for malignant extrinsic ureteric obstruction, we reported a patency rate of $86.0 \%$ at 6 months and $60.0 \%$ at 1 year among 52 patients (66 ureters). ${ }^{14}$ Our current retrospective analysis follows up on these initial findings, demonstrating the possible superiority of metallic stents over polymeric stents for the treatment of MUO. Although this advantage was not observed in multivariate analysis, we still propose that metallic stents should be the first treatment choice because of their coiled wire structure, which is superior to that of polymeric stents for maintaining patency, even under high external compression. In patients with metallic stents indwelled, there were a number of cases of bilateral ureteral obstruction due to gastrointestinal cancer, which may have influenced this outcome. We previously reported that the rate of bilateral obstruction was significantly higher than the rate of unilateral obstruction. ${ }^{14}$ Moreover, metallic stents have an anticipated indwelling period of 1 year compared to 3-6 months for polymeric stents. Although metallic stents are more expensive, it is beneficial to consider that frequent replacement is not required compared to polymeric stents.

Our rate of stent failure was low, regardless of the stent type used. MUO is caused by direct invasion of the tumor, lymph node metastasis, and abdominal dissemination. Park et $\mathrm{al}^{22}$ and Kamiyama et $\mathrm{a}^{23}$ previously reported on the decreased effectiveness of polymeric ureteral stents in cases of disseminated cancer, such as in the case of gastric tumors which disturb ureteral peristalsis. Chow et al ${ }^{13}$ investigated radiological features as possible risk factors for metallic stent failure in a relatively large cohort, providing evidence that abdominal ureteral obstruction and lymphatic metastasis around the ureter were associated with shorter functional duration. We also assessed the risk factors associated with stent failure in our study, with no clinical factors identified as being predictive of stent failure. Based on our results, ureteral stent indwelling can be safely used for MUO to secure renal function, regardless of a patient's health status.

Table 2 Results of the Cox proportional stepwise multivariate analysis of the association between the variables and stent failure

\begin{tabular}{|c|c|c|c|c|c|c|c|c|}
\hline \multirow[t]{2}{*}{ Comparison } & \multicolumn{4}{|c|}{ Unadjusted } & \multicolumn{4}{|c|}{ Adjusted } \\
\hline & HR & & & $p$-value & HR & & & $p$-value \\
\hline \multicolumn{9}{|l|}{ Cause of obstruction } \\
\hline Dissemination vs other & 1.240 & 0.609 & 2.523 & 0.553 & & & & \\
\hline Lymph node vs other & 1.156 & 0.537 & 2.488 & 0.711 & & & & \\
\hline Tumor vs other & 0.691 & 0.339 & 1.404 & 0.307 & & & & \\
\hline \multicolumn{9}{|l|}{ Stent type } \\
\hline Polymeric vs metallic & 2.153 & 1.070 & 4.230 & 0.031 & 2.032 & 0.980 & 4.210 & 0.057 \\
\hline
\end{tabular}




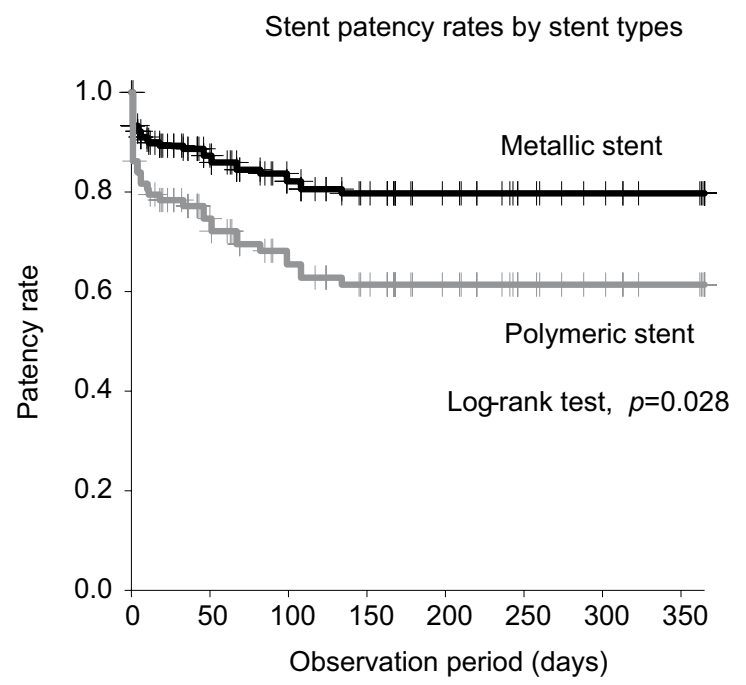

$\begin{array}{lllllllll}\begin{array}{l}\text { No. at risk } \\ \text { (metallic) }\end{array} & 72 & 52 & 41 & 35 & 24 & 19 & 15 & 11 \\ \text { No. at risk } & 54 & 36 & 31 & 28 & 24 & 22 & 20 & 16\end{array}$

Figure 3 Patency rate for the 2 stent types.

However, there are several problems with using indwelling ureteral stents for urinary tract management, especially for patients who require long-term treatment. el-Faqih et al ${ }^{24}$ reported that incrustation occurred in $9.2 \%$ of stents retrieved at 6 weeks after indwelling, with this rate increasing to $47.5 \%$ at $6-12$ weeks, and $76.3 \%$ at $>12$ weeks. Kawahara et $\mathrm{a}^{25}$ also reported encrusting in $47.0 \%$ of their implanted stents, with an encrustation rate of $26.8 \%$ at $<6$ weeks, $56.9 \%$ at $6-12$ weeks, and $75.9 \%$ at $>12$ weeks. Based on this evidence, it is clinically recommended that polymeric stents be replaced every 3-6 months. In our own case series, replacement of the indwelled polymeric stent was required in 14 of the 35 patients (40\%) treated with this stent type, over their survival period or the 1-year censored observation period. Replacement of the metallic stent was performed in 6 patients (10.5\%), after an indwelling period of $>1$ year, with no evidence of adhesion of calculi in these cases, which is not likely to adhere to metal. A noteworthy complication of indwelling stents is the development of an arterioureteral fistula, a rare but life-threatening condition. In their case series of 139 patients, van den Bergh et $\mathrm{al}^{26}$ reported that $13 \%$ of their patients died due to an arterioureteral fistula-related complication. We did not observe occurrence of arterioureteral fistula in our study cohort over the 1-year observation period. Long-term observation will be necessary to evaluate biocompatibility and observe ureteral artery fistula formation.

This study is a retrospective study, and thus has certain limitations. We could not compare between the stent-related complications of metallic and polymeric stents. Complications of an indwelling metallic stent include symptoms of bladder irritation and macrohematuria, which may affect a patient's quality of life. In this study, 4 cases (7.0\%) of gross hematuria were observed as complications after stent placement, and symptoms of bladder irritation were confirmed in 2 cases $(3.5 \%)$, without any need of anticholinergic medication. However, complications of polymeric stent cases could not be accumulated. Further studies are needed to clarify these aspects by using the ureteric stent symptom questionnaire prospectively.

\section{Conclusion}

Indwelling ureteral stents, particularly metallic stents, are effective for the treatment of MUO.

\section{Acknowledgment}

We would like to thank Editage (www.editage.jp) for English language editing.

\section{Author contributions}

All authors contributed toward data analysis, drafting and critically revising the paper and agree to be accountable for all aspects of the work.

\section{Disclosure}

The authors report no conflicts of interest in this work.

\section{References}

1. Jeong IG, Han KS, Joung JY, Seo HK, Chung J. The outcome with ureteric stents for managing non-urological malignant ureteric obstruction. BJU Int. 2007;100(6):1288-1291.

2. Wong LM, Cleeve LK, Milner AD, Pitman AG. Malignant ureteral obstruction: outcomes after intervention. Have things changed? J Urol. 2007;178(1):178-183; discussion 183

3. Elsamra SE, Leavitt DA, Motato HA, et al. Stenting for malignant ureteral obstruction: tandem, metal or metal-mesh stents. Int J Urol. 2015;22(7):629-636.

4. Sountoulides P, Kaplan A, Kaufmann OG, Sofikitis N. Current status of metal stents for managing malignant ureteric obstruction. BJU Int. 2010;105(8):1066-1072.

5. Chow PM, Chiang IN, Chen CY, et al. Malignant ureteral obstruction: functional duration of metallic versus polymeric ureteral stents. PLoS One. 2015;10(8): $\mathrm{e} 0135566$.

6. Liatsikos EN, Karnabatidis D, Katsanos K, et al. Ureteral metal stents: 10-year experience with malignant ureteral obstruction treatment. J Urol. 2009;182(6):2613-2617.

7. Borin JF, Melamud O, Clayman RV. Initial experience with full-length metal stent to relieve malignant ureteral obstruction. J Endourol. 2006;20(5):300-304.

8. Wah TM, Irving HC, Cartledge J. Initial experience with the resonance metallic stent for antegrade ureteric stenting. Cardiovasc Intervent Radiol. 2007;30(4):705-710.

9. Nagele U, Kuczyk MA, Horstmann M, et al. Initial clinical experience with full-length metal ureteral stents for obstructive ureteral stenosis. World J Urol. 2008;26(3):257-262. 
10. Benson AD, Taylor ER, Schwartz BF. Metal ureteral stent for benign and malignant ureteral obstruction. J Urol. 2011;185(6):2217-2222.

11. Wang HJ, Lee TY, Luo HL, et al. Application of resonance metallic stents for ureteral obstruction. BJU Int. 2011;108(3):428-432.

12. Goldsmith ZG, Wang AJ, Banez LL, et al. Outcomes of metallic stents for malignant ureteral obstruction. $J$ Urol. 2012;188(3):851-855.

13. Chow PM, Hsu JS, Wang SM, Yu HJ, Pu YS, Liu KL. Metallic ureteral stents in malignant ureteral obstruction: short-term results and radiological features predicting stent failure in patients with non-urological malignancies. World J Urol. 2014;32(3):729-736.

14. Asakawa J, Iguchi T, Tamada S, et al. Outcomes of indwelling metallic stents for malignant extrinsic ureteral obstruction. Int $J$ Urol. 2018;25(3):258-262.

15. Chung HH, Kim MD, Won JY, et al. Multicenter experience of the newly designed covered metallic ureteral stent for malignant ureteral occlusion: comparison with double $\mathrm{J}$ stent insertion. Cardiovasc Intervent Radiol. 2014;37(2):463-470.

16. Ellenbogen PH, Scheible FW, Talner LB, Leopold GR. Sensitivity of gray scale ultrasound in detecting urinary tract obstruction. AJR Am J Roentgenol. 1978;130(4):731-733.

17. Docimo SG, Dewolf WC. High failure rate of indwelling ureteral stents in patients with extrinsic obstruction: experience at 2 institutions. $J$ Urol. 1989;142(2 Pt 1):277-279.

18. Shekarriz B, Shekarriz H, Upadhyay J, et al. Outcome of palliative urinary diversion in the treatment of advanced malignancies. Cancer. 1999;85(4):998-1003.
19. Yossepowitch O, Lifshitz DA, DekelY, et al. Predicting the success of retrograde stenting for managing ureteral obstruction. J Urol. 2001;166(5): 1746-1749.

20. Chitale SV, Scott-Barrett S, Ho ET, Burgess NA. The management of ureteric obstruction secondary to malignant pelvic disease. Clin Radiol. 2002;57(12):1118-1121.

21. Chung SY, Stein RJ, Landsittel D, et al. 15-year experience with the management of extrinsic ureteral obstruction with indwelling ureteral stents. J Urol. 2004;172(2):592-595.

22. Park DS, Park JH, Lee YT. Percutaneous nephrostomy versus indwelling ureteral stents in patients with bilateral nongenitourinary malignant extrinsic obstruction. J Endourol. 2002;16(3):153-154.

23. Kamiyama Y, Matsuura S, Kato M, et al. Stent failure in the management of malignant extrinsic ureteral obstruction: risk factors. Int J Urol. 2011;18(5):379-382.

24. el-Faqih SR, Shamsuddin AB, Chakrabarti A, et al. Polyurethane internal ureteral stents in treatment of stone patients: morbidity related to indwelling times. J Urol. 1991;146(6):1487-1491.

25. Kawahara T, Ito H, Terao H, Yoshida M, Matsuzaki J. Ureteral stent encrustation, incrustation, and coloring: morbidity related to indwelling times. J Endourol. 2012;26(2):178-182.

26. van den Bergh RC, Moll FL, de Vries JP, Lock TM. Arterioureteral fistulas: unusual suspects-systematic review of 139 cases. Urology. 2009;74(2):251-255
Cancer Management and Research

\section{Publish your work in this journal}

Cancer Management and Research is an international, peer-reviewed open access journal focusing on cancer research and the optimal use of preventative and integrated treatment interventions to achieve improved outcomes, enhanced survival and quality of life for the cancer patient. The manuscript management system is completely online and includes
Dovepress

a very quick and fair peer-review system, which is all easy to use. Visit http://www.dovepress.com/testimonials.php to read real quotes from published authors. 